MATEC Web of Conferences 22,02011 (2015)

DOI: $10.1051 /$ matecconf/ 20152202011

(c) Owned by the authors, published by EDP Sciences, 2015

\title{
Research on the Winding Losses Based on Finite Element Method for High Frequency Transformer
}

\author{
Yongqiang Wang \& Zhihong Zheng \\ Hebei Provincial Key Laboratory of Power Transmission Equipment Security Defense, North China Electric \\ Power University, Baoding, Hebei, China
}

\begin{abstract}
The transformer loss is the important factor influencing the transformer operation. The high frequency eddy effects including skin and proximity effects raise the winding losses. The eddy current effects are calculated by the finite element method in consideration of proximity effect and skin effect. The conclusion that changing winding layout can decrease the winding losses is given, and the tendency of the losses caused by skin effect and proximity effect based on different winding thickness, frequency and layer spacing is concluded.
\end{abstract}

Keywords: high-frequency transformer; proximity effect; skin effect; FEM; winding losses

\section{INTRODUCTION}

In recent years, the switching power supply due to its high efficiency and high power density advantages has been widely used. With the miniaturization of the switching power supply, the power frequency is also increasing, and some manufacturers have accomplished frequency of $1 \mathrm{MHZ}$. Different from the power frequency transformer, the skin effect and the proximity effect will make winding loss increase rapidly based on the condition of high frequency. Thus, we can't use an analysis method of power frequency transformer winding losses to study the high frequency transformer winding losses.

Many domestic and foreign scholars have done a lot of research work for the high frequency transformer winding losses. Literature [7] presented a winding loss calculation in one-dimensional model, which makes the calculation of high frequency transformer winding loss possible. The literatures [8-11] analyzed the frequency transformer winding loss by using finite element method based on 2D condition. Literature [12-13] studied the influence of the proximity effect and the skin effect on the winding losses based on different frequency and winding layout. There are a number of scholars do research work on the magnetic components running based on the effect of high frequency ${ }^{[14-15]}$. However, these methods that have a lot of model simplifications on establishment are not able to fully reflect the real transformer winding losses.

The increasing developments of computer technology make the application of finite element method (FEM) 3D eddy current field simulation calculation for the transformer possible. Compared with other methods, the three-dimensional finite element simulation can reflect the real situation; the calculated value is more accurate. In this paper, we analyze the winding losses based on different winding thickness, frequency and layer spacing.

\section{MATHEMATICAL MODEL OF 3D EDDY CURRENT FIELD}

\subsection{Electromagnetic field equations}

Seemingly stable magnetic field can be used to solve the Maxwell equations, and the Maxwell equations in differential form are shown as follows:

$\nabla \times H=J+\frac{\partial D}{\partial t}$

$\nabla \times E=-\frac{\partial B}{\partial t}$

$\nabla \cdot B=0$

$\nabla \cdot D=\rho$

For seemingly stable magnetic field, the displacement current $\frac{\partial D}{\partial t}$ is negligible. That is to say, in the study of the seemingly stable magnetic field, we only consider the changes of magnetic field without considering the magnetic field generated by the electric field.

In the 3D eddy current field analysis, we will divide the field into the vortex zone and the non-vortex zone. We will consider the electric field and the magnetic field in the vortex zone, but in the non-vortex we will only consider the magnetic field.

In this paper, we choose the control equation $A \square \Phi$ method to establish the control equations of the electromagnetic field, and the control equations of the vortex area is shown as follows:

$\nabla \times v \nabla / A^{*}-\nabla \times v \nabla \cdot A^{*}+\sigma \frac{\partial A^{*}}{\partial t}=J$

$B=\nabla \times A^{*}$ 


$$
E=-\frac{\partial A^{*}}{\partial t}
$$

The control equations of the non-vortex area are shown as follows:

$\nabla \cdot(-\mu \nabla \psi)=0$

$\mathrm{H}=-\nabla \psi$

\subsection{Uniqueness problem}

In order to ensure that the model has a unique solution based on the given initial conditions, the eddy current field boundary of the $A, A \square \phi$ can be described in the vortex zone as follows:

$\left.\begin{array}{l}\nabla \times(v \nabla \times A)-\nabla(v \nabla \cdot A)+\sigma \frac{\partial A}{\partial t}+\sigma \nabla \phi=0 \\ \nabla \cdot\left(-\sigma \frac{\partial A}{\partial t}-\sigma \nabla \phi\right)=0\end{array}\right\}$

In the non- vortex zone, it is shown as follows:

$\nabla \times(\nu \nabla \times A)-\nabla(\nu \nabla \cdot A)=J_{S}$

In the interface, it is shown as follows:

$$
\left.\begin{array}{l}
A_{1}=A_{2} \\
v_{1} \nabla \cdot A_{1}=v_{2} \nabla \cdot A_{2} \\
v_{1} \nabla \times A_{1} \times n_{12}=v_{2} \nabla \times A_{2} \times n_{12} \\
n \cdot\left(-\sigma \frac{\partial A}{\partial t}-\sigma \nabla \phi\right)=0
\end{array}\right\}
$$

Outside of the solution, the region is shown as follows:

$$
\left.\begin{array}{l}
\left.\begin{array}{l}
n \times A=0 \\
v \nabla \cdot A=0
\end{array}\right\} \\
\begin{array}{l}
n \cdot A=0 \\
(\nu \nabla \times A) \times n=0
\end{array}
\end{array}\right\}
$$

\subsection{Boundary conditions}

The normal gradient of different media is equal as follows:

$$
\begin{aligned}
& n \times\left(\mathrm{E}_{1}-E_{2}\right)=0 \\
& n \times\left(\mathrm{B}_{1}-B_{2}\right)=0 \\
& n \times\left(\mathrm{H}_{1}-H_{2}\right)=0
\end{aligned}
$$

At the interface, the magnetic vector potential and electric scalar potential equation is shown as follows: $n \cdot\left(\sigma_{1} \frac{\partial A_{1}}{\partial t}+\sigma_{1} \nabla \phi_{1}-\sigma_{2} \frac{\partial A_{2}}{\partial t}-\sigma_{2} \nabla \phi_{2}\right)=0$

The expansion is shown as follows:

$\left(\sigma_{1}-\sigma_{2}\right) \frac{\partial A_{n}}{\partial t}=\left(\sigma_{2}\right)\left(\frac{\partial \phi}{\partial n}\right)_{2}-\left(\sigma_{1}\right)\left(\frac{\partial \phi}{\partial n}\right)_{1}$

\subsection{Calculation of conductor current}

After getting the dynamic magnetic vector potential, the current density in the conductor can be calculated by the equation $J=-j \omega \lambda \gamma A$. The current of the conductor can be obtained by the integral of the current density in the conductor section.

\section{WINDING SIMULATION MODEL}

In this paper, the round wire is simplified to the rectangle wire; the simplified model of windings is shown in Figure 1:

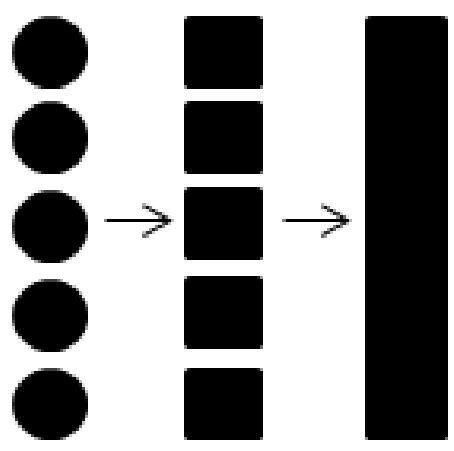

Figure 1. Simplified model of windings

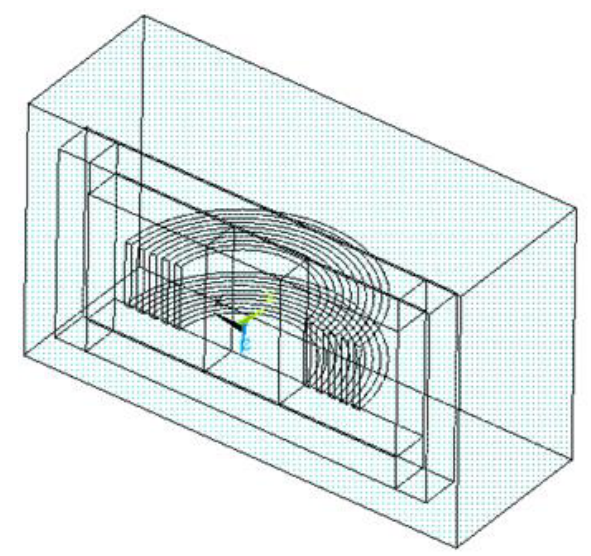


ICETA 2015

Figure 2. Three-dimensional simulation model of high fre-

In this simplified model, the circular conductor is equivalent to rectangular wire with the same height and width. The simplified model only considers the proximity effect of layers and the skin effect within layer and ignores the turn-to-turn proximity effect on the layer.

In this paper, we use E-E type transformer as the object of establishing simulation model. In order to simplify the winding model and improve the operation speed, we only establish the $1 / 2$ transformer model.

The three-dimensional simulation model of high frequency transformer is shown in Figure 2.

\section{SIMULATION CALCULATIONS}

\subsection{Influence of winding layout on the winding losses}

In order to study the transformer winding losses based on different winding layout, we designed six kinds of layouts as shown in Figure 3.

We obtain the current density distribution in winding as shown in Figure 4: quency transformer

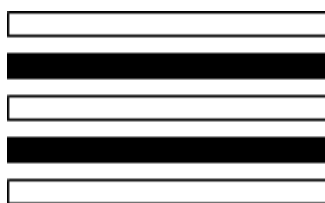

A

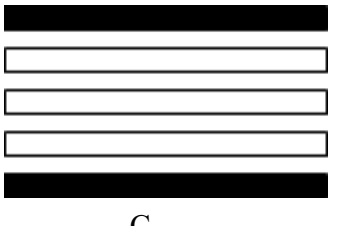

$\mathrm{C}$

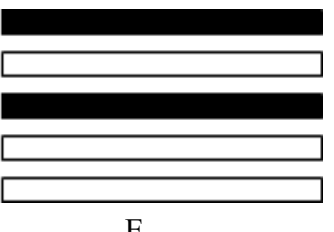

$\mathrm{E}$

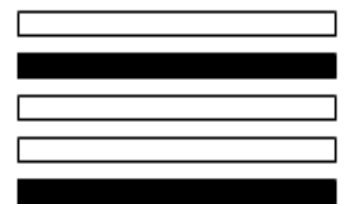

B

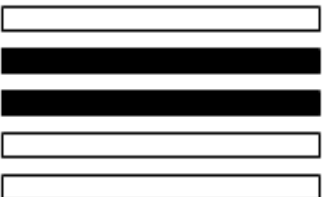

D

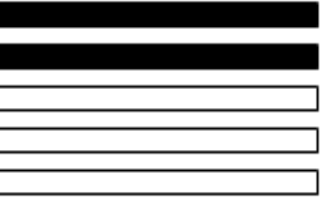

F
Figure 3. Winding structure and arrangement of transformer 
MATEC Web of Conferences

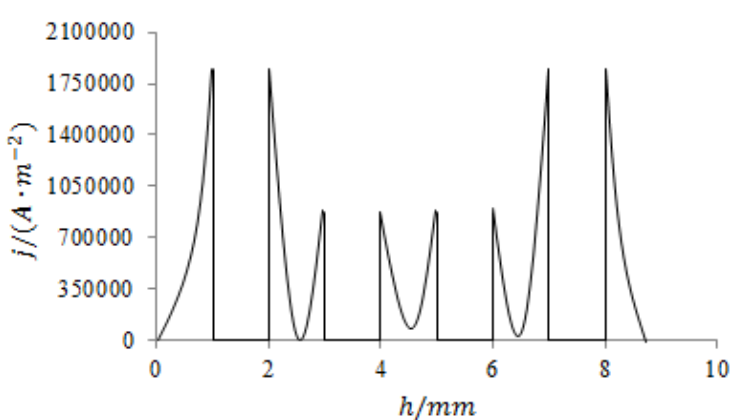

A (cross arrangement)
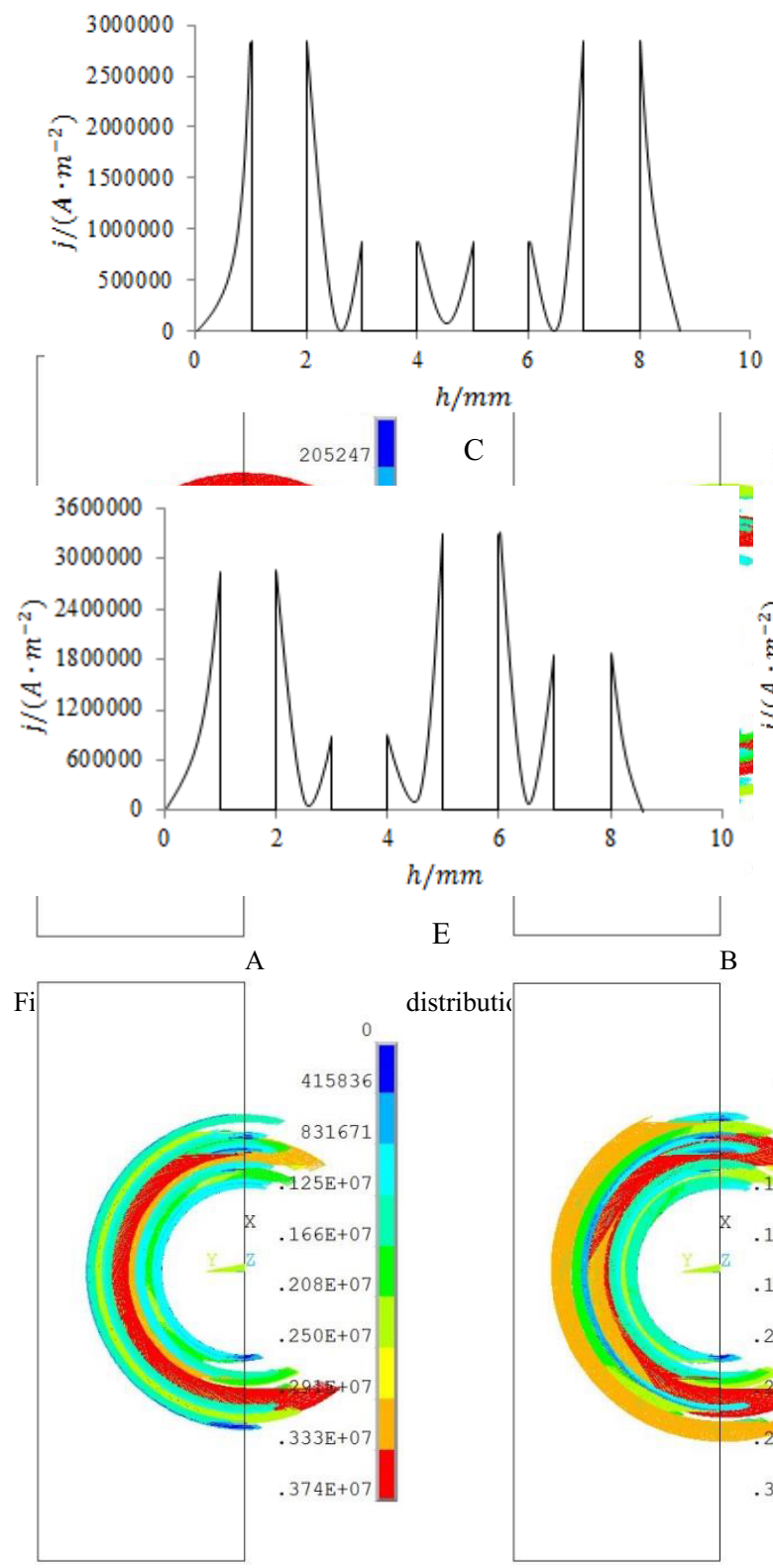

D

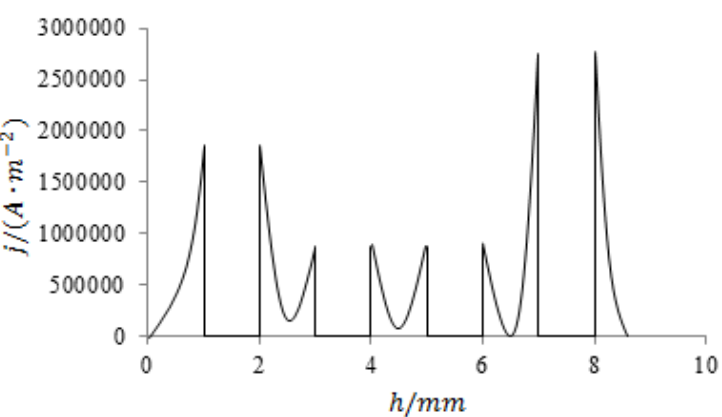

$\mathrm{B}$
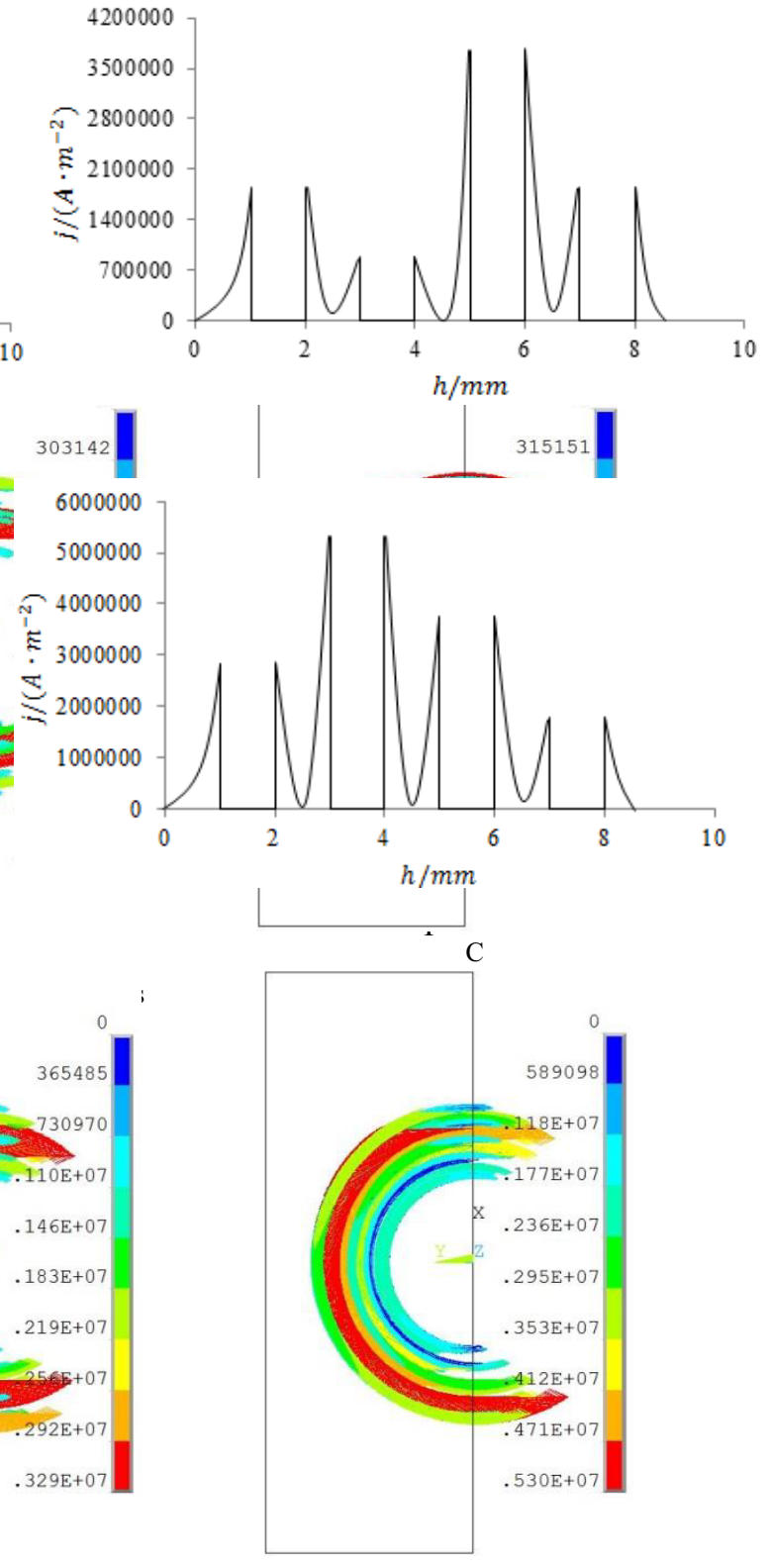

F

Figure 4. Current density distribution in windings 
We plot the curves of current density distribution for different winding structures as shown in Figure 5.

According to Figure 4, we selected three typical winding arrangements which are A (cross arrangement), $\mathrm{C}$ (sandwich arrangement) and $\mathrm{F}$ (no cross arrangement) to analyze the influence of winding layout on the winding losses.

Figure 3 and Figure 4 (F) show that when the winding arrangement is no cross layout, the winding current density is increased with the number of layers, reaching a maximum of approximately $0.530 \mathrm{E}+07$ $\mathrm{A} \cdot \mathrm{m}^{-2}$ at the interface. The winding current density started to decrease from the secondary side until reduced to zero. From the current distribution graph, we can see that this simple winding arrangement will greatly increase the winding losses. Therefore, we cannot choose this winding layout scheme in the production of transformer.

In Figure 3 and Figure 4 (C), when using the sandwich type winding arrangement, the maximum current density is about $0.284 \mathrm{E}+07 \mathrm{~A} \cdot \mathrm{m}^{-2}$.Compared with the no cross arrangement, we can see that the winding losses of sandwich arrangement are decreased by nearly half.

From Figure 3 and Figure 4 (A), it can be seen that the maximum current density is about $0.178 \mathrm{E}+0$ $\mathrm{A} \cdot \mathrm{m}^{-2}$. The winding losses of cross arrangement are decreased by nearly half. Therefore, we recommend using the cross arrangement.

Based on frequencies of $20 \mathrm{kHz}$, we calculate the winding losses of six winding layout schemes by the finite element method.

\begin{tabular}{|c|c|}
\hline Winding structures & Winding losses(W) \\
\hline A & 0.040364 \\
\hline B & 0.047538 \\
\hline $\mathrm{C}$ & 0.062668 \\
\hline $\mathrm{D}$ & 0.073368 \\
\hline E & 0.074465 \\
\hline $\mathrm{F}$ & 0.133732 \\
\hline
\end{tabular}

Compared with the cross arrangement, we can see from Table 1 that the sandwich arrangement did not have a larger increase in the winding loss. The structure of cross arrangement is complex, and the capacitance is larger. On the less demanding situations of winding losses, we recommend the use of sandwich winding.

\subsection{Influence of winding thickness on the winding losses}

In order to research the influence of winding thickness for the transformer winding losses, we choose A (cross arrangement), $\mathrm{C}$ (sandwich arrangement) and $\mathrm{F}$ (no cross arrangement) these three most typical wind- ing layouts to simulate. We study the winding losses of these three methods of winding arrangement with the winding thickness changing. The simulation results are shown in Table 2:

Table 2. Winding loss based on different winding thickness

\begin{tabular}{llll}
\multirow{2}{*}{$\begin{array}{l}\text { Thickness } \\
(\mathrm{mm})\end{array}$} & \multicolumn{2}{l}{ Winding structures } \\
\cline { 2 - 4 } & $\mathrm{A}(\mathrm{W})$ & $\mathrm{C}(\mathrm{W})$ & $\mathrm{F}(\mathrm{W})$ \\
0.2 & 0.1472 & 0.1316 & 0.1259 \\
0.3 & 0.1131 & 0.0966 & 0.0899 \\
0.4 & 0.0928 & 0.0739 & 0.0688 \\
0.5 & 0.0917 & 0.0649 & 0.0557 \\
0.6 & 0.0914 & 0.0609 & 0.0503 \\
0.7 & 0.0981 & 0.0612 & 0.0452 \\
0.8 & 0.1095 & 0.0619 & 0.0432 \\
0.9 & 0.1125 & 0.0616 & 0.0421 \\
1 & 0.1121 & 0.0617 & 0.0402 \\
1.1 & 0.1123 & 0.0612 & 0.0403 \\
1.2 & 0.1472 & 0.1316 & 0.1259 \\
1.3 & 0.1131 & 0.0966 & 0.0899 \\
\hline
\end{tabular}

From Table 2, at the beginning with increasing the thickness of the winding, we can see the winding losses decreases rapidly. However, when the winding thickness is increased to a certain extent, the decrease of amplitude of winding loss began to slow until the winding loss is basically fixed.

When the winding thickness is small, A (cross), C (sandwich arrangement) and $\mathrm{F}$ ((no cross arrangement) winding loss of these three kinds of layouts are not of much difference. That is to say, when the winding thickness is small, the winding arrangement for winding losses had little effect. But when the winding thickness began to increase, the loss of different winding arrangements began to have significant change. From Table 3, we can see the change of winding thickness for A (cross arrangement) with the greatest impacts, for $\mathrm{C}$ (sandwich arrangement) with less impacts than that for $\mathrm{A}$, and for $\mathrm{F}$ (no cross arrangement) with minimum impacts.

\subsection{Influence of frequency on the winding losses}

In order to research the influence of frequency for the transformer winding losses, we choose A (cross arrangement), $\mathrm{C}$ (sandwich arrangement) and $\mathrm{F}$ (no cross arrangement) these three most typical winding layouts to simulate. We study the winding losses of these three methods of winding arrangement with frequency changes. The simulation results are shown in Table 3:

Table 3. Winding loss based on different frequency 
MATEC Web of Conferences

\begin{tabular}{|c|c|c|c|c|c|c|c|}
\hline \multirow{2}{*}{$\begin{array}{l}\text { Frequency } \\
(\mathrm{kHZ})\end{array}$} & \multicolumn{3}{|c|}{ Winding structures } & 0.9 & 0.04040 & 0.06847 & 0.14131 \\
\hline & $\mathrm{A}(\mathrm{W})$ & $\mathrm{C}(\mathrm{W})$ & $\mathrm{F}(\mathrm{W})$ & 1.1 & 0.04129 & 0.06911 & 0.14141 \\
\hline 0 & 0.0012 & 0.0011 & 0.0016 & 1.2 & 0.04219 & 0.06962 & 0.14152 \\
\hline 20 & 0.0403 & 0.0652 & 0.1334 & 1.3 & 0.04301 & 0.07017 & 0.14011 \\
\hline 40 & 0.0589 & 0.1113 & 0.2716 & 1.4 & 0.04331 & 0.07051 & 0.14035 \\
\hline
\end{tabular}

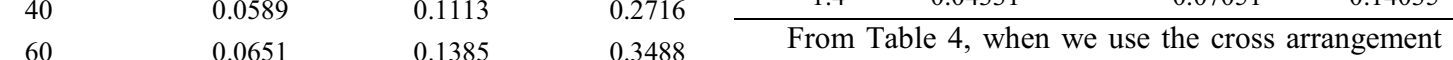

0.3488 and sandwiches arrangement, the winding losses in-

$80 \quad 0.0789 \quad 0.1572 \quad 0.3955 \quad$ crease a little with the layer spacing. And when we use

$100 \quad 0.0907 \quad 0.1714 \quad 0.4265 \quad$ no cross arrangement, the winding losses decrease a

$120 \quad 0.1011 \quad 0.1822 \quad 0.4489 \quad$ little with layer spacing. Overall, the influence of the

$1400.1113 \quad 0.1909 \quad 0.4662$ distance between layers on winding losses is very

weak, and we ean almost ignore it. Therefore, in the

$160 \quad 0.1197 \quad 0.1982 \quad 0.4799 \quad \begin{aligned} & \text { weak, and we can almost ignore it. Therefore, in the } \\ & \text { production of high-frequency transformer, we do not }\end{aligned}$

$180-0.1276-0.2039 \quad 0.4912-$ productic

$200 \quad 0.1358 \quad 0.2088$ have too much to consider the impact of interlayer

spacing for the winding losses. How to select the

$0.2131 \quad 0.5091$ layer spacing depends on the Leakage inductance, the

We can see from Table 8 that when it is increased with the frequency at the beginning, the winding losses decrease rapidly. However, with the increase of frequency, the growth rate of winding loss begins to be slow until it reaches a stable growth trend.

When the frequency is small, the winding losses of three winding arrangements have little difference. However, with the increase of frequency, F (no cross arrangement) loss increases fastest, $\mathrm{C}$ (sandwich arrangement) is slower than $\mathrm{F}$ and $\mathrm{A}$ (cross) is the slowest. Thus, we can know the influence of frequency on the three kinds of arrangements is $F$ (no cross arrangement) $>\mathrm{C}$ (sandwich arrangement) $>\mathrm{A}$ (cross arrangement). The higher the frequency is, the more obvious influence of winding layout for the winding loss is.

4.4 Influence of winding layer spacing on the winding losses

We study on the influence of winding layer spacing on the winding losses by changing the three winding layout layer spacing. Results are shown in Table 4:

Table 4. Winding loss based on different layer spacing

\begin{tabular}{|c|c|c|c|}
\hline \multirow{2}{*}{$\begin{array}{l}\text { Layer } \\
\text { spacing } \\
(\mathrm{mm})\end{array}$} & \multicolumn{3}{|c|}{ Winding structures } \\
\hline & $\mathrm{A}(\mathrm{W})$ & $\mathrm{C}(\mathrm{W})$ & $\mathrm{F}(\mathrm{W})$ \\
\hline 0.2 & 0.03661 & 0.06616 & 0.15486 \\
\hline 0.3 & 0.03692 & 0.006637 & 0.15231 \\
\hline 0.4 & 0.03763 & 0.06656 & 0.15039 \\
\hline 0.5 & 0.03798 & 0.06701 & 0.15001 \\
\hline 0.6 & 0.03869 & 0.06727 & 0.14724 \\
\hline 0.7 & 0.03912 & 0.06754 & 0.14701 \\
\hline 0.8 & 0.03978 & 0.06799 & 0.14533 \\
\hline
\end{tabular}

\section{CONCLUSIONS}

(1) The amplitude of the current in the windings can be effectively reduced when the winding is adjacently arranged to the different current flow. By comparing the $\mathrm{C}$ (sandwich arrangement) and A (cross arrangement) winding losses in the two layout arrangements, we find that compared with the cross arrangement and the sandwich arrangement, the winding losses did not have obvious increase. The cross arrangement is complex, and the capacitance is larger.

(2) With increasing the thickness of the winding at the beginning, the winding losses decrease rapidly. However, when the winding thickness is increased to a certain extent, the speed of decreasing amplitude of winding losses begins to be slow until the winding loss is basically fixed. When the winding thickness is small, the influence of winding arrangements for the losses is not strong. But when the thickness is increased, the loss of significant changes is in. we can see the change of winding thickness for A (cross arrangement) with the greatest impacts, for $\mathrm{C}$ (sandwich arrangement) with less impacts and $\mathrm{F}$ (no cross arrangement) with minimum impacts.

(3) The influence of frequency on these three kinds of arrangement is $\mathrm{F}$ (no cross arrangement) $>\mathrm{C}$ (sandwich arrangement) $>\mathrm{A}$ (cross).

(4) The effect of winding layer spacing for the winding loss is very limited.

\section{ACKNOWLEDGEMENT}

This project is supported by the Fundamental Research Funds for the Central Universities (13MS73) and National Key Technology Support Pro- 
gram"Research of electronic power transformer Based on the demand of lightweight (2014BJ0045)".

\section{REFERENCES}

[1] Liang Xiaoguo. Wei Jian,Ruan Xinbo. 2004. A novel interleaved three-level forward converter. Proceedings of the CSEE, 24(11): 139-143.

[2] HuZong bo. \& Zhang Bo.2002. Study on bi-directional conductibility and power loss of power MOSFET in synchronous rectifier. Proceedings of the CSEE, 22(3):88-93.

[3] Zhang Haiyuan, Wu Hui. \& Zou Zubing, et al. 2003.Modeling and analysis of symmetrical half bridge current-doubler converter with synchronous rectifier applied. Proceedings of the CSEE, 23(8): 66-70.

[4] Qian Zhaoming. \& He Xiangning, 1997. The recent developments of power electronics and its applications (I) proceedings of the CSEE, 17(6):

[5] Qian Zhaoming, Dong Bofan. \& He Xiangning.1998. The recent developments of power electronics and its applications (II). Proceedings of the CSEE, 18(3): 153-162.

[6] Ren Xiaoyong. \& Ruan Xinbo. 2005. Two-stage converter applicable to high voltage input low voltage output case. Proceedings of the CSEE, 25(23): 153-157.

[7] Dowell P. 1966. Effect of eddy currents in transformer windings. IEEProc, 113(8): 1387-1394.

[8] Prieto R, Cobos J A. \& Garía O, et al.1997. Interleaving techniques in magnetic components. Proc. IEEE APEC, Atlanta, USA: 931 -936.

[9] Ning Dai. \& Lee F C. 1997.High-frequency eddy-current effects in low- profile transformer windings. Proc. IEEE PESC, Saint Louis, USA: 641 -647.

[10] Abed N Y. \& Mohammed O A. 2010. Physics-based high frequency transformer modeling by finite elements. IEEE Transactions on Magnetics, 46(8): 3249-3252.

[11][Susa D. \& Nordman H.2009. A simple model for calculating transformer hot-spot temperature. IEEE Transactions on Power Delivery, 24(3): 1257-1265.

[12] Ferreira J A. 1990. Appropriate modelling of conductive losses in the design of magnetic components. Proc. IEEE PESC, San Antonio, USA: 780-785.

[13]Ferreira J A.1994. Improved analytical modeling of conductive losses in magnetic components.IEEE Trans.on Power Electronics, 9(1):127-131.

[14]Kuang Jianj un Ruan Xinbo. \& Ren Xiaoyong.2005. Consideration for current sharing among parallel windings in planar transformers. Proceedings of the CSEE, 25(14):146-150.

[15]Dai N,Lofti A W, Skutt G,et al. 1994.A comparative study of high frequency, low-profile planar transformer technologie s. Proc. IEEE APEC, Orlando, USA: 226-232. 